\title{
Overexpression of carbohydrate sulfotransferase 15 in pancreatic cancer stroma is associated with worse prognosis
}

\author{
YOKO MATSUDA $^{1,2}$, YUKO FUJII ${ }^{3}$, MIHO MATSUKAWA ${ }^{3}$, TOSHIYUKI ISHIWATA ${ }^{4}$, \\ MAKOTO NISHIMURA $^{3}$ and TOMIO ARAI ${ }^{1}$ \\ ${ }^{1}$ Department of Pathology, Tokyo Metropolitan Geriatric Hospital, Itabashi, Tokyo 173-0015; \\ ${ }^{2}$ Oncology Pathology, Department of Pathology and Host-Defense, Faculty of Medicine, Kagawa University, \\ Kita, Kagawa 761-0793; ${ }^{3}$ Department of Endoscopy, Tokyo Metropolitan Geriatric Hospital; \\ ${ }^{4}$ Tokyo Metropolitan Geriatric Hospital and Institute of Gerontology, Itabashi, Tokyo 173-0015, Japan
}

Received April 12, 2019; Accepted July 11, 2019

DOI: $10.3892 / 01.2019 .10764$

\begin{abstract}
Carbohydrate sulfotransferase 15 (CHST15) synthesizes matrix proteoglycan that regulates various pathogenic mediators and contributes to tissue remodeling and fibrosis during injury. CHST15 has been reported to promote tumor growth and invasion in various types of cancer. Our previous study reported the safety and efficacy of EUS-guided fine-needle injection (EUS-FNI) of STNM01, a double-stranded RNA oligonucleotide that specifically represses CHST15, for use in patients with pancreatic cancer. The present study aimed to determine the expression and clinicopathological characteristics of CHST15 in pancreatic cancer. Immunohistochemical staining was performed for CHST15 using pancreatic tissues from 64 patients ( 28 males and 36 females; age, $69.0 \pm 9.6$ years) with pancreatic cancer who underwent surgery. For the evaluation of fibrosis, two categories were defined (mature and immature), based on the existence of collagen, myxoid stroma and fibroblasts, using hematoxylin and eosin specimens. The positive percentage of CHST15 was quantified, patients were divided into two groups according to high and low CHST15 expression in both the cancer and stroma tissues, and the association between CHST15 expression in cancer cells and the stroma was analyzed. Additionally, the present study analyzed the association between CHST15 expression and clinicopathological information, including overall and disease-free survival. The expression levels of CHST15 were detected in the cytoplasm of pancreatic cancer cells and fibroblasts in the cancer stroma. CHST15 expression in cancer cells was not identified to be associated with overall survival $(\mathrm{P}=0.52)$.
\end{abstract}

Correspondence to: Dr Yoko Matsuda, Oncology Pathology, Department of Pathology and Host-Defense, Faculty of Medicine, Kagawa University, 1750-1 Ikenobe, Miki-cho, Kita, Kagawa 761-0793, Japan

E-mail: youkoh@med.kagawa-u.ac.jp

Key words: carbohydrate sulfotransferase 15, pancreatic cancer, molecular targeting therapy, fibrosis
However, patients with high CHST15 expression in the stroma exhibited worse overall survival compared with patients with low CHST15 expression ( $\mathrm{P}=0.02)$. CHST15 expression in the stroma exhibited a positive association with that in cancer cells $(\mathrm{P}=0.01)$. High CHST15 expression in the stroma group was associated with a higher incidence of immature fibrosis $(\mathrm{P}=0.02)$ compared with mature fibrosis. CHST15 expression in cancer cells was associated with Union for International Cancer Control stage $(\mathrm{P}=0.02)$ and invasive front. Age and sex were not associated with CHST15 expression. The present study revealed that overexpression of CHST15 in stroma was associated with worse overall survival and immature fibrosis. Overexpression of CHST15 in cancer cells was associated with tumor stage. These results suggested that targeting therapy for CHST15 may be useful for stroma fibroblasts and cancer cells.

\section{Introduction}

Fibrosis is the formation of excess fibrous connective tissue and deposition of extracellular matrix by fibroblasts. Fibrosis can be a reactive, benign, healing process; nonetheless, in cancer tissues, fibrous stroma can form a part of the cancer microenvironment, providing favorable conditions for tumor progression (1). Fibrosis represents one of the pathological characteristics of pancreatic cancer, a cancer that often shows significant fibrosis with low vascularization. Fibrous tissue supports cancer cell progression and causes chemoresistance, which possibly leads to the poor prognosis of pancreatic cancer. Therefore, fibroblast-targeting therapy has been considered an important goal in pancreatic cancer treatment (2).

Various stromal proteoglycans have been reported to play critical roles in cancer progression and are paid attention as important modulators of tumor microenvironment $(3,4)$. Carbohydrate sulfotransferase 15 (CHST15) has been reported to contribute to tissue remodeling during injury and fibrosis (5), and promote tumor growth and invasion (6). CHST15 selectively synthesizes matrix proteoglycan, chondroitin sulfate $\mathrm{E}$ (CS-E), which cleaves CD44 on pancreatic cancer cells and thus is involved in cancer invasion (7). In this respect, blockade of CHST 15 by siRNA was shown to improve tissue remodeling in 
the lung, esophagus, colon and heart in animal models (5,8-10). STNM01, the double-stranded RNA oligonucleotide that specifically inhibits CHST15 expression, repressed pre-existing fibrosis in Crohn's disease patients $(11,12)$. For pancreatic cancer, siRNA targeting CHST15 suppressed cell proliferation in vitro and inhibited tumor growth in a subcutaneous xenograft tumor model in mice (6).

Ito et al (3) reported that CHST15 overexpression in tumor tissue was associated with poor overall survival and disease-free survival in pancreatic cancer. Although the authors did not show which cell types were positive for CHST15, it is suggested that CHST15 would be an emerging target for pancreatic cancer (3). However, the expression and function of CHST15 in stromal fibroblasts in pancreatic tumor tissue have not been reported yet. The aim of the present study was to determine the expression and clinicopathological characteristics of CHST15 in pancreatic cancer, with special focus on its relation to fibrosis.

\section{Materials and methods}

Patients and tissue samples. To determine the expression of CHST15 in pancreatic cancer, we retrospectively prepared resected pancreatic tissues from 64 patients (28 males and

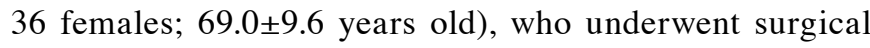
treatment between 1988 and 2013. All patients suffered from invasive ductal adenocarcinoma, and none received neoadjuvant chemoradiotherapy. Pathological specimens were analyzed by pathologists, based on the World Health Organization Classification of Tumours of the Digestive System (13). We collected clinical information from medical records. UICC stage was determined based on the pathological diagnosis. Overall survival was determined from the date of surgery to mortality from any cause. Disease-free survival was determined as the date of surgery to the date of recurrence of pancreatic cancer. The present study was conducted in accordance with the principles embodied in the Declaration of Helsinki, 2013, and all experiments were approved by the ethics committee of the Tokyo Metropolitan Geriatric Hospital (permit no. R16-09). Informed written consent to use the tissues was obtained from all patients.

Immunohistochemistry. The largest cross-sectional slice was used for immunohistochemical staining. Paraffin-embedded sections (3- $\mu \mathrm{m}$ thick) were subjected to immunostaining and hematoxylin and eosin (H\&E) staining. After deparaffinization, the tissue sections were preheated in HEAT PROCESSOR Solution pH 6 (Nichirei Corporation, Tokyo, Japan) for $20 \mathrm{~min}$ at $100^{\circ} \mathrm{C}$. Sections were incubated for $5 \mathrm{~min}$ at room temperature with Protein Block Serum-Free (Agilent Technologies, CA). Then, the sections were incubated with mouse monoclonal anti-CHST15 antibody (diluted at 1:50, HPA017584, Merck KGaA, Darmstadt, Germany). Afterwards, the sections were incubated with EnVision FLEX RABBIT (Agilent Technologies). Bound antibodies were detected with diaminobenzidine tetrahydrochloride as the substrate, using Histofine Simple Stain MAX PO (Nichirei Corporation). The sections were then counterstained with Mayer's hematoxylin. Negative control tissue sections were prepared by incubation without the primary antibody.
Evaluation of tissue specimens. We evaluated the positive percentage of cancer cells and stroma, because CHST15 expression in stroma might play important roles in cancer progression. CHST15 expression was evaluated in five random fields within the tumor under a light microscope (magnification, $\mathrm{x} 200$ ). The percentage of positive cancer cells and stroma cells for CHST15 was evaluated separately. Immunohistochemical review was performed by two of the authors (Yoko Matsuda and Toshiyuki Ishiwata), who were blinded to clinical and outcome data, with a mean value of two being used for statistical analysis. We divided the patients into groups using a cutoff value $40 \%$ according to the median value of CHST15 expression in cancer cells.

For evaluation, we classified fibrosis into two categories (mature and immature), based on the existence of collagen, myxoid stroma, or fibroblasts (1) using H\&E specimens. Immature fibrosis can be defined as an amorphous stromal substance composed of an amphophilic or slightly basophilic material usually combined with randomly oriented collagen fibers (Fig. 1A). Mature fibrosis can be defined as fine collagen fibers stratified into multilayers, with few fibroblasts, and sometimes containing eosinophilic hyalinization (Fig. 1B).

Statistical analysis. Results are presented as the mean \pm standard deviation (SD). Differences between the two groups were compared using the Mann-Whitney U test. Chi-square tests were used to analyze clinicopathological features. Kaplan-Meier analysis and the log-rank test were used to examine the association with overall survival. The correlation between cancer and stromal CHST15 expression was analyzed by Pearson's correlation coefficient. A P-value of $<0.05$ was considered significant in all analyses. All statistical analyses were performed using the StatView J version 5.0 software package (SAS Institute, Inc., Cary, NC, USA), and Excel (Office 2010, Microsoft Corporation, Redmond, WA, USA).

\section{Results}

CHST15 expression in the pancreas. The expression of CHST15 was detected in the cytoplasm of pancreatic cancer cells (Fig. 2A, arrows and inset) and fibroblasts in the cancer stroma (Fig. 2B, arrow and inset). Furthermore, there was a strong expression of CHST15 in cancer cells in the invasive front (Fig. 2C and D) when compared with that in the center area of the cancer (Fig. 2E). Some cases did not express CHST15 in cancer cells, while stroma cells expressed CHST15 (Fig. 2F, arrows). In non-cancerous tissue, centroacinar cells (arrows and inset), pancreatic duct epithelium (arrowheads), and islets (asterisks) expressed CHST15 (Fig. 2G).

Survival and CHST15 expression. We divided the patients into two groups according to CHST15 expression in each of the cancer cells and stroma using a $40 \%$ cutoff. CHST15 expression in cancer cells was not associated with overall survival ( $\mathrm{P}=0.52$, Fig. 3A). However, the group showing high-CHST15 expression in cancer stroma showed worse overall survival than the low-CHST15 expression group ( $\mathrm{P}=0.02$, Fig. 3B). CHST15 expression in cancer cells was not associated with disease-free survival ( $\mathrm{P}=0.85$, Fig. $3 \mathrm{C}$ ), while the group showing high CHST15 expression in the cancer stroma showed a shorter 

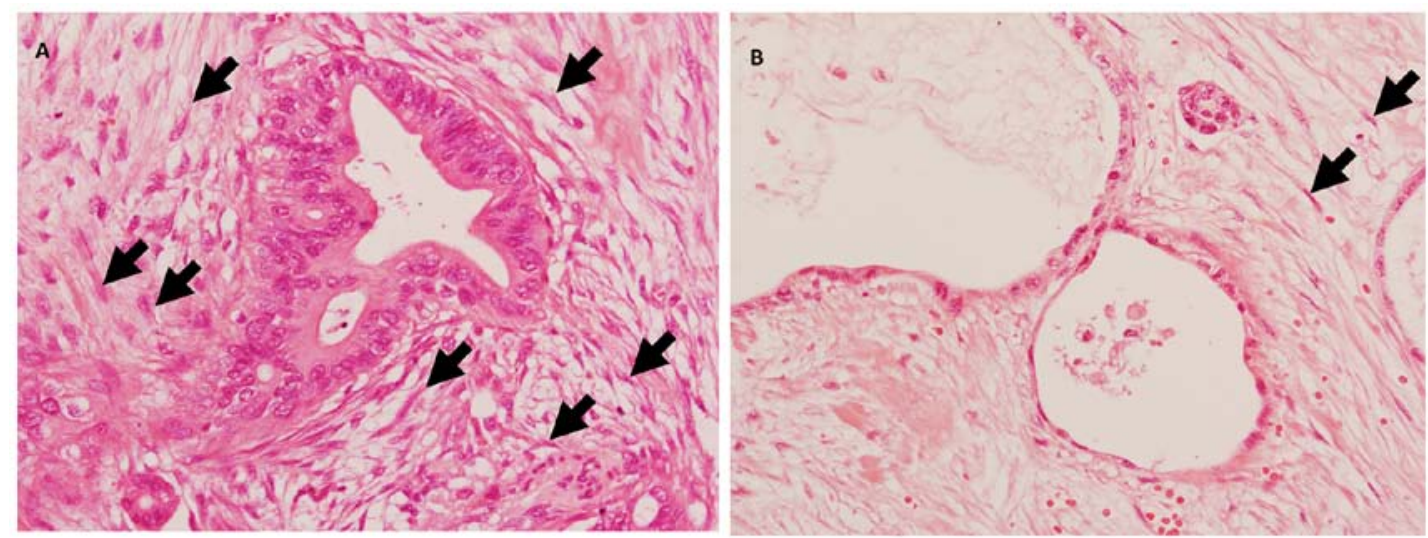

Figure 1. Fibrous pattern of pancreatic cancer. (A) Immature fibrosis contains an amorphous stromal substance composed of an amphophilic material intermingled with randomly oriented collagen fibers. (B) Mature fibrosis contains fine collagen fibers stratified into multilayers with eosinophilic hyalinization, and few fibroblasts. Arrows indicate fibroblasts. Hematoxylin and eosin stain. Original magnification, $\mathrm{x} 400$.

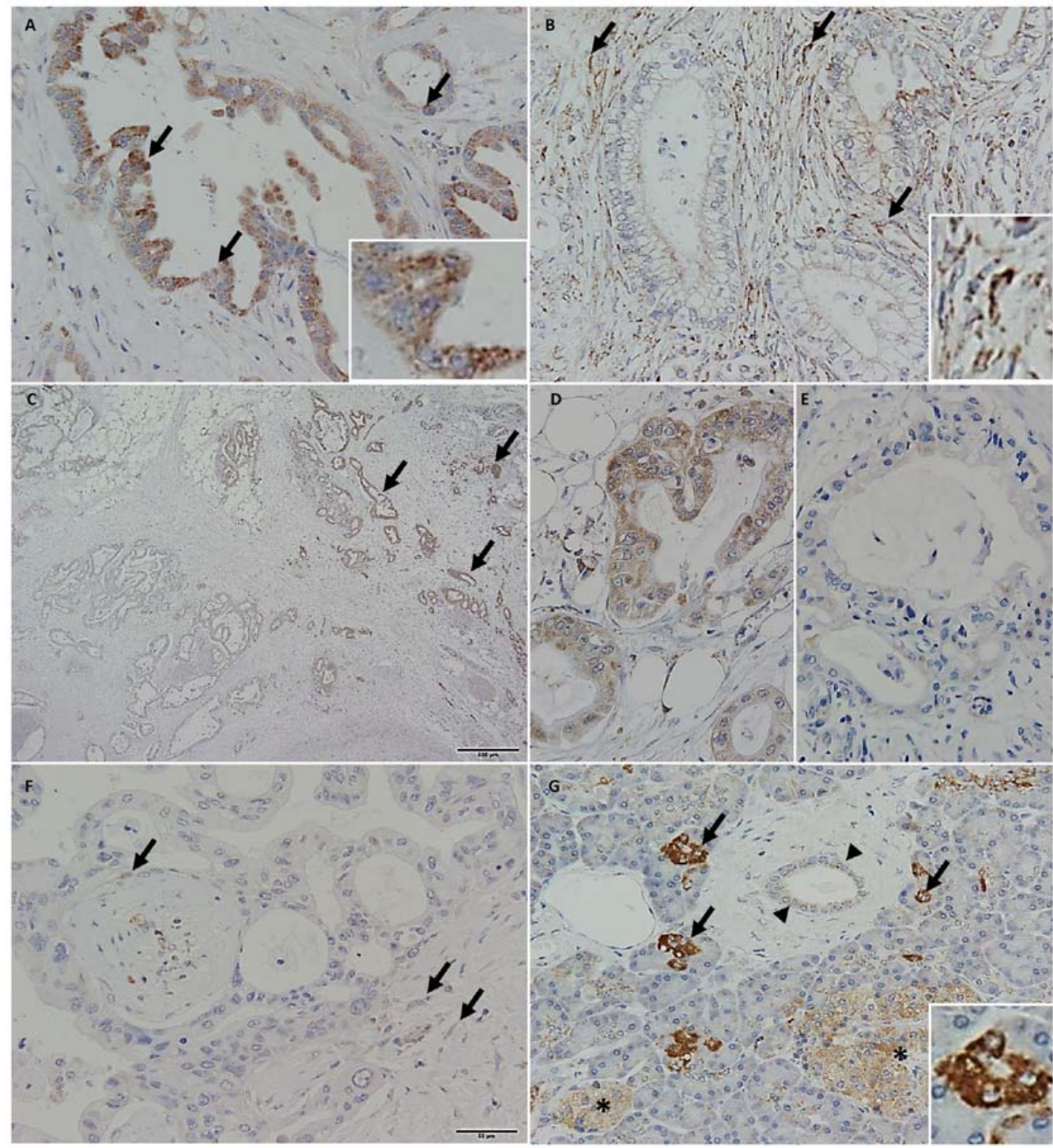

Figure 2. Expression levels of CHST15 in the pancreas. CHST15 was expressed in the cytoplasm of (A) pancreatic cancer cells (arrows and inset) and (B) fibroblasts (arrows and inset). CHST15 expression in the invasive front of pancreatic cancer. (C) CHST15 was strongly expressed in the invasive front of the cancer (arrows) compared with that in the central area of the cancer. Corresponding images from (D) the invasive front and (E) the center area. (F) Pancreatic cancer cases were negative for CHST15 in cancer cells and positive for CHST15 in stroma cells (arrows indicate stroma cells). (G) CHST15 was expressed in centroacinar cells (arrows and inset), duct epithelium (arrowheads) and islets (asterisks). Original magnification, x40 (C), x200 (G) and x400 (A, B, D, E and F). CHST15, carbohydrate sulfotransferase 15 . 

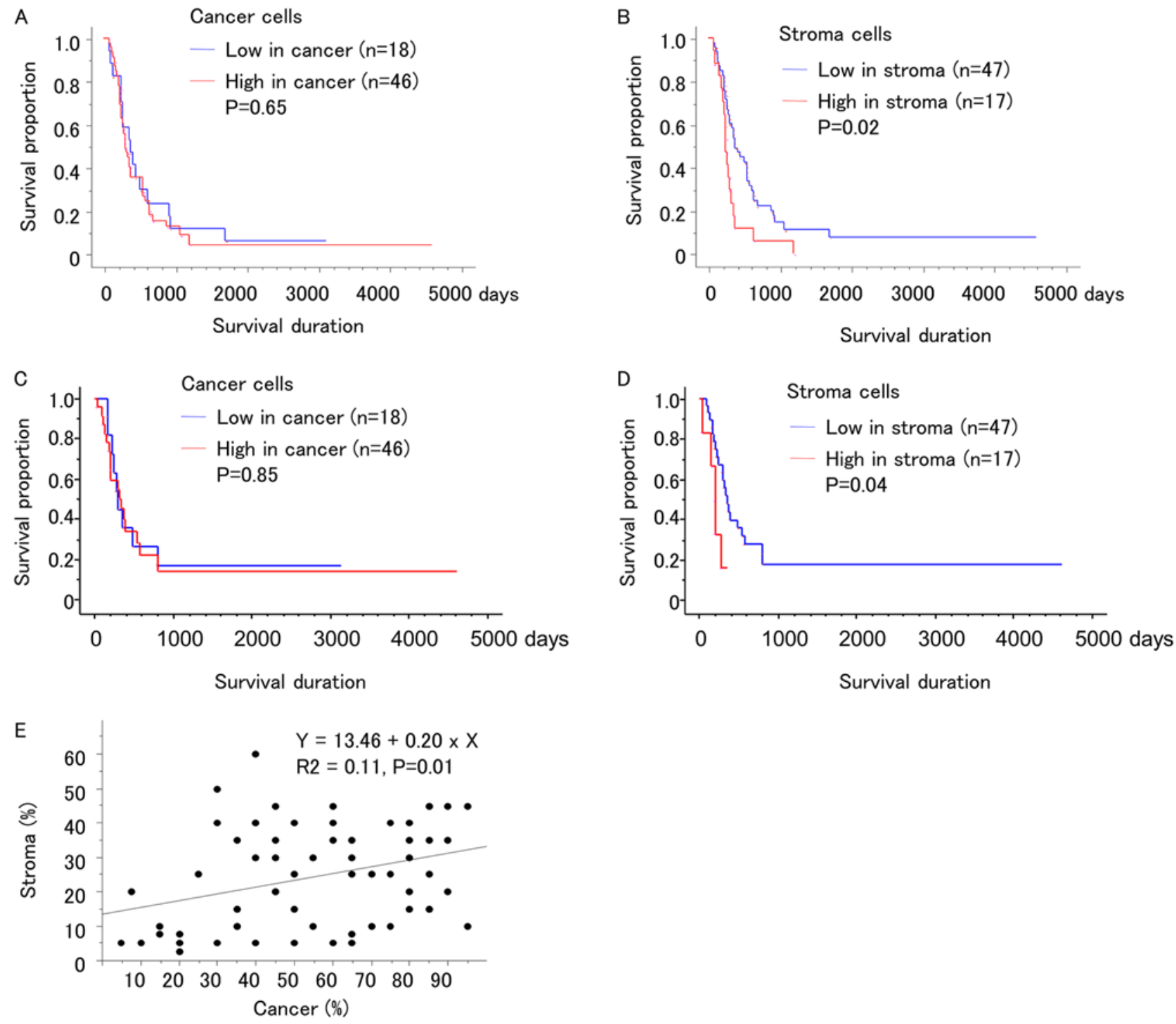

Figure 3. CHST15 expression and prognosis of pancreatic cancer. (A) Overall survival and expression of CHST15 in cancer cells. (B) Overall survival and expression of CHST15 in stroma. (C) Disease free survival and expression of CHST15 in cancer cells. (D) Disease free survival and expression of CHST15 in stroma. (E) CHST15 expression in cancer cells exhibited a positive correlation with that in stroma. CHST15, carbohydrate sulfotransferase 15.

disease-free survival than the low CHST15 expression group $(\mathrm{P}=0.04$, Fig. 3D). The expression of CHST15 in cancer cells showed a positive correlation with that in the stroma (Fig. 3E).

Clinicopathological characteristics of CHST15 expression. Age and gender were not associated with CHST15 expression (Table I). In fibrous patterns, high CHST15 expression in stroma cells was associated with immature fibrosis $(\mathrm{P}=0.02)$. CHST15 expression in cancer cells was associated with UICC stage $(\mathrm{P}=0.02)$.

\section{Discussion}

The present study revealed that expression of CHST15 in pancreatic cancer stroma was associated with disease prognosis. The cases with overexpression of CHST15 in the stroma showed worse overall survival than the cases with low expression of CHST15 in the stroma, suggesting the effectiveness of CHST15 as a therapeutic target.
Ito et al (3) observed a relationship between CHST15 expression and prognosis in 36 patients with pancreatic ductal adenocarcinoma. They analyzed CHST15 expression in five random fields within the tumor by light microscopy. They graded CHST15 staining statuses as follows: 1, Negative, no staining or little staining in $\leq 20 \%$ of pancreatic ductal adenocarcinoma tissue; 2 , Weak, weak staining in $>80 \%$ of pancreatic ductal adenocarcinoma (PDA) tissue; 3, Moderate, moderate staining in $>80 \%$ of PDA tissue; and 4 , Strong, strong or complete staining in $>80 \%$ of PDA tissue. When they compared tissues with scores 4 vs scores $1-3$, CHST15 overexpression was found to be associated with overall survival and disease-free survival. However, they did not distinguish CHST15 expression between the cancer cells and stroma. Furthermore, it is difficult to evaluate the intensity of immunohistochemical staining; therefore, we focused on the percentage of positive cells to evaluate CHST15 expression. This is the first study to show the localization and characteristics of CHST15 in pancreatic cancer. Our study clearly 
Table I. Expression of CHST15 and clinicopathological characteristics.

\begin{tabular}{|c|c|c|c|c|c|c|}
\hline Variables & Low in cancer cells & High in cancer cells & P-value & Low in stroma & High in stroma & P-value \\
\hline Cases, $\mathrm{n}$ & 18 & 46 & & 47 & 17 & \\
\hline \multicolumn{7}{|l|}{$\begin{array}{l}\text { Percentage of CHST15 } \\
\text { expression, mean } \pm \text { SD }\end{array}$} \\
\hline Cancer cells & $22.9 \pm 10.7$ & $66.0 \pm 17.5$ & $<0.01^{\mathrm{a}}$ & $50.6 \pm 25.4$ & $62.9 \pm 22.6$ & 0.08 \\
\hline Stroma & $15.1 \pm 13.8$ & $27.4 \pm 13.9$ & $<0.01^{\mathrm{a}}$ & $17.2 \pm 10.6$ & $42.6 \pm 6.4$ & $<0.01^{\mathrm{a}}$ \\
\hline Age, years $($ mean $\pm S D)$ & $67.1 \pm 8.6$ & $69.7 \pm 10.0$ & 0.34 & $69.1 \pm 9.1$ & $68.6 \pm 11.3$ & 0.88 \\
\hline \multicolumn{7}{|l|}{ Sex, n (\%) } \\
\hline Male & $7(38.9)$ & $21(45.7)$ & 0.62 & $22(46.8)$ & $6(35.3)$ & 0.41 \\
\hline Female & $11(61.1)$ & $25(54.3)$ & & $25(53.2)$ & $11(64.7)$ & \\
\hline \multicolumn{7}{|l|}{ Differentiation, n (\%) } \\
\hline Well & $11(61.1)$ & $19(41.3)$ & 0.35 & $24(51.2)$ & $6(35.3)$ & 0.34 \\
\hline Moderate & $6(33.3)$ & $22(47.8)$ & & $18(38.3)$ & $10(58.8)$ & \\
\hline Poor & $1(5.6)$ & $5(10.9)$ & & $5(10.6)$ & $1(5.9)$ & \\
\hline \multicolumn{7}{|l|}{ Fibrosis pattern, n (\%) } \\
\hline Mature fibrosis & $12(66.7)$ & $32(69.6)$ & 0.82 & $36(76.6)$ & $8(47.1)$ & $0.02^{\mathrm{a}}$ \\
\hline Immature fibrosis & $6(33.3)$ & $14(30.4)$ & & $11(23.4)$ & $9(52.9)$ & \\
\hline \multicolumn{7}{|l|}{ UICC stage, n (\%) } \\
\hline I & $0(0)$ & $3(6.5)$ & $0.02^{\mathrm{a}}$ & $3(6.4)$ & $0(0)$ & 0.35 \\
\hline II & $14(77.8)$ & $42(91.3)$ & & $39(83.0)$ & $17(100)$ & \\
\hline III & $3(16.7)$ & $0(0)$ & & $3(6.4)$ & $0(0)$ & \\
\hline IV & $1(5.6)$ & $1(2.2)$ & & $2(4.3)$ & $0(0)$ & \\
\hline
\end{tabular}

${ }^{\mathrm{a}} \mathrm{P}<0.05$. Mann-Whitney $\mathrm{U}$ and $\chi^{2}$ tests were used for statistical analysis. CHST15, carbohydrate sulfotransferase 15; UICC, Union for International Cancer Control, 8th Edition.

revealed that stromal CHST15 expression is correlated with prognosis.

Stromal CHST15 expression was associated with the fibrous pattern of pancreatic cancer. Cases with low CHST15 expression in stroma showed hyalinized stroma with few fibroblasts (mature fibrosis), while those with high CHST15 expression showed active fibrosis with abundant fibroblasts (immature fibrosis). This suggests that CHST15 in stroma regulates fibroblasts and fibrosis, which is consistent with previous reports $(5,8-10)$. Additionally, CHST15 may be related to fibroblast activation. Cancer-associated fibroblasts can provide favorable conditions for pancreatic cancer progression, which makes CHST15 targeting therapy useful in decreasing the number of cancer-associated fibroblasts.

CHST15 in cancer cells was correlated with invasive front. Suzuki et al (9) showed that the expression of CHST15 was associated to epithelial-mesenchymal transition in vitro study. There is a possibility that CHST15's function in cancer cells is different from that in stromal fibroblasts; thus, CHST15 targeting therapy might affect both stroma fibroblasts and cancer cells. The absolute area of CHST15-positive cancer cells was higher than that of CHST15-positive fibroblasts. Additionally, a positive correlation was observed between cancer and stromal expression of CHST15, suggesting common mechanisms of regulation of CHST15 in cancer cells and stroma cells. CHST15 expression showed large variations in stromal and cancer; therefore, the OS of cancer and stromal CHST15 expression was different. Based on the results of UICC stage, CHST15 expression in cancer cells may be associated with an earlier stage. Further analysis is needed to clarify the molecular functions of CHST15 gene mutation or methylation, or protein glycosylation or phosphorylation.

Recently we reported on the safety of CHST15-targeted therapy in patients with pancreatic cancer by echo ultrasound-guided fine needle injection (14). The effectiveness of CHST15 targeting therapy in high-CHST15 compared with that in low-CHST patients needs to be determined. CHST15 immunostaining might be a useful method to predict the therapeutic effects of CHST15 targeting therapy. Neoadjuvant chemoradiotherapy for pancreatic cancer often induces significant fibrosis (15); therefore, CHST15 targeting therapy has the possibility to improve prognosis for neoadjuvant patients.

We also detected expression of CHST15 in centroacinar cells and islets in the pancreas. Centroacinar cells have been considered the origin of pancreatic cancer as well as the source of pancreatic stem cells (16). These cells play important roles in carcinogenesis and tissue repair during injury. There are currently no reports regarding the expression and roles of CHST15 in centroacinar cells and islets; however, these findings might affect the side effects of CHST15 targeting therapy.

The present study has several limitations. Ito et al (3) determined the relationship between CHST15 expression and overall (duration from the time of diagnosis)/disease-free survival (duration from the date of surgery), while we examined the overall and disease-survival from the time of surgery. Further studies using different cohort including healthy control 
and unresectable pancreatic cancer will be needed to establish CHST15 targeting therapy.

In conclusion, CHST15 in cancer stroma is associated with fibrous pattern and prognosis. Further studies are required to implement CHST15 targeting therapy in pancreatic cancer.

\section{Acknowledgements}

Not applicable.

\section{Funding}

The present study was supported in part by a grant-in-aid from the Japan Society for the Promotion of Science (grant no. 16KT0125) and a grant for translational research from Tokyo Metropolitan Geriatric Hospital and Institute of Gerontology to YM.

\section{Availability of data and materials}

All data analyzed during this study are included in this published article.

\section{Authors' contributions}

YM, MN and TA conceived and directed the project, analyzed data, and wrote the manuscript. YM and TI evaluated immunohistochemical specimens. YF and MM analyzed clinical data. All authors read and approved the final manuscript.

\section{Ethics approval and consent to participate}

The present study was conducted in accordance with the principles embodied in the Declaration of Helsinki, 2013, and all experiments were approved by the Ethics Committee of the Tokyo Metropolitan Geriatric Hospital (approval no. R16-09). Informed written consent to use the tissues was obtained from all patients.

\section{Patient consent for publication}

Not applicable.

\section{Competing interests}

The authors declare that they have no competing interests.

\section{References}

1. Ueno H, Shinto E, Shimazaki H, Kajiwara Y, Sueyama T, Yamamoto J and Hase K: Histologic categorization of desmoplastic reaction: Its relevance to the colorectal cancer microenvironment and prognosis. Ann Surg Oncol 22: 1504-1512, 2015.

2. Katz MH, Fleming JB, Bhosale P, Varadhachary G, Lee JE, Wolff R, Wang H, Abbruzzese J, Pisters PW, Vauthey JN, et al: Response of borderline resectable pancreatic cancer to neoadjuvant therapy is not reflected by radiographic indicators. Cancer 118: 5749-5756, 2012.
3. Ito Z, Takakura K, Suka M, Kanai T, Saito R, Fujioka S, Kajihara M, Yanagisawa H, Misawa T, Akiba T, et al: Prognostic impact of carbohydrate sulfotransferase 15 in patients with pancreatic ductal adenocarcinoma. Oncol Lett 13: 4799-4805, 2017.

4. Yamamoto T, Matsuda Y, Kawahara K, Ishiwata T and Naito Z: Secreted $70 \mathrm{kDa}$ lumican stimulates growth and inhibits invasion of human pancreatic cancer. Cancer Lett 320: 31-39, 2012.

5. Kai Y, Tomoda K, Yoneyama H, Kitabatake M, Nakamura A, Ito T, Yoshikawa $\mathrm{M}$ and Kimura $\mathrm{H}$ : Silencing of carbohydrate sulfotransferase 15 hinders murine pulmonary fibrosis development. Mol Ther Nucleic Acids 6: 163-172, 2017.

6. Takakura K, Shibazaki Y, Yoneyama H, Fujii M, Hashiguchi T, Ito Z, Kajihara M, Misawa T, Homma S, Ohkusa T and Koido S: Inhibition of cell proliferation and growth of pancreatic cancer by silencing of carbohydrate sulfotransferase 15 in vitro and in a xenograft model. PLoS One 10: e0142981, 2015.

7. Sugahara KN,Hirata T, Tanaka T, Ogino S, Takeda M, Terasawa H, Shimada I, Tamura J, ten Dam GB, van Kuppevelt TH and Miyasaka M: Chondroitin sulfate E fragments enhance CD44 cleavage and CD44-dependent motility in tumor cells. Cancer Res 68: 7191-7199, 2008.

8. Sato H, Sagara S, Nakajima N, Akimoto T, Suzuki K, Yoneyama H, Terai S and Yahagi N: Prevention of esophageal stricture after endoscopic submucosal dissection using RNA-based silencing of carbohydrate sulfotransferase 15 in a porcine model. Endoscopy 49: 491-497, 2017.

9. Suzuki K, Arumugam S, Yokoyama J, Kawauchi Y, Honda Y, Sato H, Aoyagi Y, Terai S, Okazaki K, Suzuki Y, et al: Pivotal role of carbohydrate sulfotransferase 15 in fibrosis and mucosal healing in mouse colitis. PLoS One 11: e0158967, 2016.

10. Watanabe K, Arumugam S, Sreedhar R, Thandavarayan RA, Nakamura T, Nakamura M, Harima M, Yoneyama $H$ and Suzuki K: Small interfering RNA therapy against carbohydrate sulfotransferase 15 inhibits cardiac remodeling in rats with dilated cardiomyopathy. Cell Signal 27: 1517-1524, 2015.

11. Suzuki K and Yoneyama H: New endoscopic approach of anti-fibrotic therapy for inflammatory bowel disease. Ann Transl Med 5: 191, 2017.

12. Suzuki K, Yokoyama J, Kawauchi Y, Honda Y, Sato H, Aoyagi Y, Terai S, Okazaki K, Suzuki Y, Sameshima Y, et al: Phase 1 clinical study of siRNA targeting carbohydrate sulphotransferase 15 in crohn's disease patients with active mucosal lesions. J Crohns Colitis 11: 221-228, 2017.

13. Hruban RH, Boffetta P, Hiraoka N, Iacobuzio-Donahue C, Kato Y, Kern SE, et al: Tumours of the pancreas. In: Bosman FT, Carneiro F, Hruban RH, Theise ND (eds). WHO Classification of Tumours of the Digestive System. 4th edition. IARC, Lyon, 2010.

14. Nishimura M, Matsukawa M, Fujii Y, Matsuda Y, Arai T, Ochiai Y, Itoi T and Yahagi N: Effects of EUS-guided intratumoral injection of oligonucleotide STNM01 on tumor growth, histology, and overall survival in patients with unresectable pancreatic cancer. Gastrointest Endosc 87: 1126-1131, 2018.

15. Miyashita T, Tajima H, Makino I, Okazaki M, Yamaguchi T, Ohbatake Y, Nakanuma S, Hayashi H, Takamura H, Ninomiya I, et al: Neoadjuvant chemotherapy with gemcitabine plus nab-paclitaxel reduces the number of cancer-associated fibroblasts through depletion of pancreatic stroma. Anticancer Res 38: 337-343, 2018.

16. Zhou ZC, Dong QG, Fu DL, Gong YY and Ni QX: Characteristics of Notch2(+) pancreatic cancer stem-like cells and the relationship with centroacinar cells. Cell Biol Int 37: 805-811, 2013.

This work is licensed under a Creative Commons Attribution-NonCommercial-NoDerivatives 4.0 International (CC BY-NC-ND 4.0) License. 